INPLASY

PROTOCOL

To cite: Sun et al. Efficacy of the combination of teriparatide and denosumab in the treatment of postmenopausal osteoporosis. Inplasy protocol 202210092. doi:

10.37766/inplasy2022.1.0092

Received: 18 January 2022

Published: 18 January 2022

Corresponding author: Yang Sun

sunyang.rocket@outlook.com

Author Affiliation:

the First Hospital of Jilin

University.

Support: Natural Science

Foundation.

Review Stage at time of this submission: Preliminary searches.

Conflicts of interest:

None declared.

\section{Efficacy of the combination of teriparatide and denosumab in the treatment of postmenopausal osteoporosis}

Sun, Y1; Li, Y².

Review question / Objective: The patients are women with postmenopausal osteoporosis. The intervention is a combination of teriparatide and denosumab. Controls were denosumab or teriparatide applied alone. The outcomes mainly include bone mineral density (BMD), fractures, bone turnover markers and adverse events. Data are extracted entirely from randomized controlled trials (RCTs).

Condition being studied: Postmenopausal osteoporosis, resulting from oestrogen deficiency, is the most common type of osteoporosis. Oestrogen deficiency results in an increase in bone turnover owing to effects on all types of bone cells. The imbalance in bone formation and resorption has effects on trabecular bone (loss of connectivity) and cortical bone (cortical thinning and porosity). The incidence of fractures varies greatly by country, but on average up to $50 \%$ of women $>50$ years of age are at risk of fractures. Osteoporosis is diagnosed using bone density measurements of the lumbar spine and proximal femur. Preventive strategies to improve bone health include diet, exercise and abstaining from smoking. Fractures may be prevented by reducing falls in high-risk populations. Several drugs are licensed to reduce fracture risk by slowing down bone resorption (such as denosumab) or by stimulating bone formation (such as teriparatide).Treatment options for postmenopausal osteoporosis are still being explored.

INPLASY registration number: This protocol was registered with the International Platform of Registered Systematic Review and Meta-Analysis Protocols (INPLASY) on 18 January 2022 and was last updated on 18 January 2022 (registration number INPLASY202210092).

\section{INTRODUCTION}

Review question / Objective: The patients are women with postmenopausal osteoporosis. The intervention is a combination of teriparatide and denosumab. Controls were denosumab or teriparatide applied alone. The outcomes 
mainly include bone mineral density (BMD), fractures, bone turnover markers and adverse events. Data are extracted entirely from randomized controlled trials (RCTs).

Condition being studied: Postmenopausal osteoporosis, resulting from oestrogen deficiency, is the most common type of osteoporosis. Oestrogen deficiency results in an increase in bone turnover owing to effects on all types of bone cells. The imbalance in bone formation and resorption has effects on trabecular bone (loss of connectivity) and cortical bone (cortical thinning and porosity). The incidence of fractures varies greatly by country, but on average up to $50 \%$ of women $>50$ years of age are at risk of fractures. Osteoporosis is diagnosed using bone density measurements of the lumbar spine and proximal femur. Preventive strategies to improve bone health include diet, exercise and abstaining from smoking. Fractures may be prevented by reducing falls in high-risk populations. Several drugs are licensed to reduce fracture risk by slowing down bone resorption (such as denosumab) or by stimulating bone formation (such as teriparatide).Treatment options for postmenopausal osteoporosis are still being explored.

\section{METHODS}

Participant or population: Participants were women with postmenopausal osteoporosis. Subjects were excluded if they had evidence of hyperparathyroidism, vitamin D deficiency, and if they had ever taken parental bisphosphonates, and osteoporosis was due to causes other than postmenopausal.

Intervention: Detail of inclusion: administration with combination of teriparatide and denosumab. Teriparatide is a synthetic form of the natural human parathyroid hormone. This medicine helps your body to form new bone, increase bone mineral density and bone strength. Studies have shown that subcutaneous teriparatide is effective in women with postmenopausal osteoporosis.Denosumab (Prolia) is a human monoclonal antibody targeting the key bone resorption mediator RANKL. The drug is administered via subcutaneous injection once every 6 months and is approved for various indications, including the treatment women with postmenopausal osteoporosis. Detail of exclusion:applying only one of the above two drugs or other drugs.

Comparator: Detail of inclusion: women with postmenopausal osteoporosis, using only teriparatide or denosumabDetails of exclusion:applying both teriparatide and denosumab; applying other drugs such as bisphosphonates.

Study designs to be included: Eligible RCTs.

Eligibility criteria: P: women with postmenopausal osteoporosis; I: combination of teriparatide and denosumab; $\mathrm{C}$ : denosumab or teriparatide applied alone; $\mathrm{O}$ : bone mineral density (BMD), fractures, bone turnover markers and adverse events; S: RCT.

Information sources: All of our information is sourced from database searches. The sources include MEDLINE, Embase, Web of Science, Cochrane. We have no restrictions on languages or publication dates.

Main outcome(s): Bone mineral density (BMD). In bone mineral density (BMD) scanning, the energy of $x$ ray beams that are passed through bones is absorbed, and what is not absorbed is detected on the other side of the body. The more dense the bones (from greater mineral content), the more energy is absorbed, and the less energy detected. In modern scanners, $x$ ray beams of two different energies are used (dual $x$ ray absorptiometry), giving a dose up to that of a chest $x$ ray. If the whole skeleton is being examined a higher dose is received because of the greater area exposed (about 1.5 chest $x$ rays). Two energies allow an estimate to be made for soft tissue absorption separately from that of the bones.Values for bone density may be quoted as $\mathrm{g} / \mathrm{cm} 2$ or converted into values related to the average female (or male) peak bone mass or to the bone mass 
related to the patient's age. These are $T$ scores and $Z$ scores. Osteoporosis is diagnosed if the $T$ score is over -2.5 according to the WHO (1994).Z scores may be used to monitor long term follow up of treatment.

Quality assessment / Risk of bias analysis: We use Cochrane for risk of bias (quality) assessment.

Strategy of data synthesis: We will use STATA for data synthesis. Select of fixed effects model (Mantel-Haenszel) or random effects (IV-heterogeneity) model depending on the magnitude of heterogeneity. If a random effects model is chosen, we can further implement subgroup analyses based on characteristics of the RCT such as the site of BMD measurement.

Subgroup analysis: We grouped the subjects according to the site of BMD measurement, their age and the dose of teriparatide and denosumab respectively.

Sensitivity analysis: Sensitivity analyses will be conducted on data syntheses with high heterogeneity in order to find the specific articles that contribute to the heterogeneity.

Country(ies) involved: China.

Keywords: postmenopausal osteoporosis;

teriparatide; denosumab.

Contributions of each author:

Author 1 - Yang Sun.

Email: sunyang.rocket@outlook.com

Author 2 - Yue Li.

Email: 382997395@qq.com 